\title{
Complete and assembled genome sequence of an NDM-9- and CTX-M-15-producing Klebsiella pneumoniae ST147 wastewater isolate from Switzerland
}

\author{
Nüesch-Inderbinen, Magdalena ; Zurfluh, Katrin ; Stevens, Marc J A ; Stephan, Roger
}

\begin{abstract}
OBJECTIVES: Carbapenem-resistant Klebsiella pneumoniae have emerged worldwide and represent a major threat to human health. Here we report the genome sequence of K. pneumoniae 002SK2, an NDM-9- and CTX-M-15-producing strain isolated from wastewater in Switzerland and belonging to the international high-risk clone sequence type 147 (ST147). METHODS: Whole-genome sequencing of K. pneumoniae 002SK2 was performed using Pacific Biosciences (PacBio) single-molecule, real-time (SMRT) technology RS2 reads (C4/P6 chemistry). De novo assembly was performed using Canu assembler, and sequences were annotated using the NCBI Prokaryotic Genome Annotation Pipeline (PGAP). RESULTS: The genome of K. pneumoniae 002SK2 consists of a 5.4-Mbp chromosome containing blaSHV-11 and fosA6, a 159-kb IncFIB $(\mathrm{K})$ plasmid carrying the heavy metal resistance genes ars and sil, and a 77-kb IncR plasmid containing blaCTX-M-15, blaNDM-9, blaOXA-9 and blaTEM-1. CONCLUSIONS: Multidrugresistant K. pneumoniae harbouring blaNDM-9 and blaCTX-M-15 are spreading into the environment, most probably via wastewater from clinical settings.
\end{abstract}

DOI: https://doi.org/10.1016/j.jgar.2018.03.001

Posted at the Zurich Open Repository and Archive, University of Zurich

ZORA URL: https://doi.org/10.5167/uzh-168120

Journal Article

Accepted Version

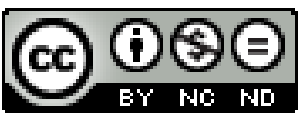

The following work is licensed under a Creative Commons: Attribution-NonCommercial-NoDerivatives 4.0 International (CC BY-NC-ND 4.0) License.

Originally published at:

Nüesch-Inderbinen, Magdalena; Zurfluh, Katrin; Stevens, Marc J A; Stephan, Roger (2018). Complete and assembled genome sequence of an NDM-9- and CTX-M-15-producing Klebsiella pneumoniae ST147 wastewater isolate from Switzerland. Journal of Global Antimicrobial Resistance, 13:53-54.

DOI: https://doi.org/10.1016/j.jgar.2018.03.001 
1 Complete and assembled genome sequence of an NDM-9 and CTX-M-15 producing

2 Klebsiella pneumoniae sequence type 147 wastewater isolate from Switzerland

3

4 Magdalena Nüesch-Inderbinen ${ }^{1 *}$, Katrin Zurfluh ${ }^{1}$, Marc J.A. Stevens ${ }^{1}$, Roger Stephan ${ }^{1}$

5

6

$7{ }^{1}$ Institute for Food Safety and Hygiene, Vetsuisse Faculty, University of Zurich, Zurich,

8 Switzerland

9

$10 *$ Corresponding author:

11 Magdalena Nüesch-Inderbinen, Institute for Food Safety and Hygiene, Vetsuisse Faculty,

12 University of Zurich, Winterthurerstrasse 272, CH-8057 Zurich, Switzerland.

13 Phone +41 4463586 51, Fax +41 4463589 08, e-mail magdalena.nüesch-

14 inderbinen@uzh.ch 


\section{Abstract}

17 Objectives: Carbapenem-resistant Klebsiella pneumoniae (CRKP) have emerged worldwide and represent a major threat to human health. Here, we report the genome sequence of $K$. pneumoniae 002SK2, an NDM-9 and CTX-M-15 producing strain isolated from wastewater

20 in Switzerland and belonging to the international high-risk clone ST147.

21 Methods: Whole genome sequencing of $K$. pneumoniae 002SK2 was performed using Pacific

22 Biosciences (PacBio) single-molecule real-time (SMRT) technology RS2 reads (C4/P6

23 chemistry). De novo assembly was performed using canu assembler, and sequences were 24 annotated using the NCBI Prokaryotic Genome Annotation Pipeline.

25 Results: The genome of $K$. pneumoniae 002SK2 consists of a 5.4-Mbp chromosome containing bla SHV-11 $_{\text {and }}$ fos $A 6$, an $\operatorname{IncFIB}(\mathrm{K})$ 159-kb plasmid carrying heavy metal resistance genes ars and sil, and an IncR 77-kb plasmid containing bla $a_{\mathrm{CTX}-\mathrm{M}-15}$, bla NDM-9, $_{\text {, }}$ blaXA-9 and bla $\mathrm{TEM}-1$.

Conclusions: Multidrug resistant $K$. pneumoniae harbouring bla $a_{\mathrm{NDM}-9}$ and bla CTX-M-15 are spreading into the environment, most probably via wastewater from clinical settings.

Keywords

K. pneumoniae $\mathrm{ST} 147$, bla $a_{\mathrm{NDM}-9}, b_{\text {CTX-M-15, }}$ genome analysis 
41 Klebsiella pneumoniae is not only ubiquitous in the environment and a common intestinal

42 commensal, but also an important human pathogen, causing both nosocomial and community

43 acquired infections. K. pneumoniae sequence type (ST)147 has emerged as a major

44 international high-risk nosocomial clone and has recently been associated with VIM-1,

45 NDM-1 and KPC-2 carbapenemases in many countries [1]. The carbapenemase NDM-9

46 differs from NDM-1 by a single amino acid substitution (E152K) and was first reported in the

47 clinical K. pneumoniae ST107 isolate PPH1303 from China in 2013, followed by its

48 detection in the mcr-1 harbouring Escherichia coli strain THJS02 and in three environmental

49 K. variicola isolates GJ1, GJ2, and GJ3 in South Korea [2].

50 K. pneumoniae isolate 002SK2 was isolated from wastewater in Basel, Switzerland in

51 December 2015 [3]. DNA extraction was performed with the Wizard $^{\circledR}$ Genomic

52 DNAPurification Kit according to the manufacturers protocol (Promega AG, Dübendorf,

53 Switzerland). The genome was sequenced at the Functional Genomics Center Zurich

54 (FGCZ), Switzerland, using two single-molecule real-time (SMRT) cells on a PacBio RS II

55 (Pacific Biosciences, Menlo Park, CA, USA). The raw reads were filtered using the RS Filter

56 Only protocol in the SMRT-portal (Pacific Biosciences) with 0.85 as polymerase read quality

57 cut off and a minimal length of $500 \mathrm{bp}$. A total of 120,283 reads with an average length of

$5812,411 \mathrm{bp}$ were selected, corresponding to $1,492,829,662$ sequenced basepairs and a genome

59 coverage of approximately 280 fold. The reads were assembled using the Canu assembler [4]

60 with the option "-pacbio-raw" and an estimated genome size of 5.4 Mbp. The Canu output

61 consisted of 4 contigs which were further polished in CLC workbench 7 (CLC, Aarhus,

62 Denmark). The chromosomal origin of replication was identified using DoriC 5.0 and

63 plasmid origins of replications were determined by PlasmidFinder 1.3. The genome was

64 annotated by the NCBI Prokaryotic Genomes Automatic Annotation Pipeline (GPAP) server. 
The genome of $K$. pneumoniae $002 \mathrm{SK} 2$ has a $\mathrm{G}+\mathrm{C}$ content of $57.4 \%$ and consists of a 5,393,461-bp chromosome with the origin of replication situated upstream of gidA, a common feature in Klebsiella species. The start of the sequence was set at 9 bp upstream of the first DnaA box in the origin region. Two extra-chromosomal elements of 159,714 and 77,809 bp, designated p002SK2-A and p002SK2-B, respectively, were identified. The PGAP server predicted a total of 5356 protein-coding sequences.

The chromosome of $K$. pneumoniae 002SK2 contains the ß-lactam resistance gene bla $\mathrm{SHV}-11$ and the fosfomycin resistance gene fos $A 6$. The 159,714-bp plasmid p002SK2-A ( $\operatorname{IncFIB}(K))$ carries no acquired antibiotic resistance genes but contains an ars operon encoding for arsenic resistance, cop genes encoding copper resistance, and sil system components mediating silver resistance. Plasmid 77,809-bp p002SK2-B (IncR) carries genes encoding resistance to aminoglycosides (aacA4, aph(3')$V I)$, B-lactams (bla $a_{\mathrm{TEM}-1}, b_{\text {OXX-9 }}$, bla $_{\mathrm{CTX}-\mathrm{M}-15}$, and bla $\left._{\mathrm{NDM}-9)}\right)$, bleomycin $($ ble $)$, fluoroquinolones ( $q n r S)$, and rifampin (arr-2), and the mercury resistance (merc) operon. The plasmid p002SK2-B does not harbour tra genes for conjugal transfer, but contains a toxin/antitoxin (A/T) $\operatorname{VapBC}$ operon. The bla $a_{\mathrm{NDM}-9}$ gene is located in a region of $\sim 7.5 \mathrm{~kb}$ bracketed by a $172 \mathrm{bp}$ truncated insertion sequence (IS)Aba14 (100\% identity to GenBank accession number CP001921) and IS26 (Figure 1). The bla $a_{\mathrm{NDM}-9}$ gene is located downstream of ISAba125, representing a genetic arrangement highly conserved among NDM-producing Gram-negative species. ISSpu_2

85 (GenBank accession number NC_009438) transposed into the ISAba125 element at bp position 877 of the ISAba125 transposase gene (Figure 1). An antimicrobial resistance gene conferring resistance to aminoglycosides, aph(3')-VI is located upstream of ISAba125 and downstream of the truncated ISAba14. Except for the ISSpu_2 transposase region, the upstream and downstream environment of the $b l a_{\mathrm{NDM}-9}$ gene shares high similarity with that 
90

95

96

97

98

99

100

101

102

103

104

105

106

107

108

109 Ethical approval

110 Not required. 


\section{References}

113 [1] Baraniak A, Izdebski R, Fiett J, Sadowy E, Adler A, Kazma M, et al. Comparative

114 population analysis of Klebsiella pneumoniae strains with extended-spectrum $\beta$ -

115 lactamases colonizing patients in rehabilitation centers in four countries. Antimicrob

$116 \quad$ Agents Chemother 2013; 57:1992-1997.

117 [2] Di DY, Jang J, Unno T, Hur HG. Emergence of Klebsiella variicola positive for NDM-

1189 , a variant of New Delhi metallo- $\beta$-lactamase, in an urban river in South Korea. J

$119 \quad$ Antimicrob Chemother 2017; 72:1063-1067.

120 [3] Zurfluh K, Bagutti C, Brodmann P, Alt M, Schulze J, Fanning S et al. Wastewater is a 121 reservoir for clinically relevant carbapenemase- and 16s rRNA methylase-producing 122 Enterobacteriaceae. Int J Antimicrob Agents 2017; 50:436-440.

123 [4] Koren S, Walenz BP, Berlin K, Miller JR, Bergman NH, Phillippy AM. Canu: scalable 124 and accurate long-read assembly via adaptive k-mer weighting and repeat separation. 125 Genome Res 2017; 27:722-736. 


\section{Figure legend}

128 Linear map of the bla $a_{\mathrm{NDM}-9}$ encoding region of p002SK2-B. Antimicrobial resistance genes

129 are coloured in red, insertion sequences (IS) are shown in dark or light blue, other genes are 130 shown in burgundy.

$131 \operatorname{aph}\left(3^{\prime}\right)-V I$, gene coding for aminoglycoside 3'-phosphotransferase; bla $a_{\mathrm{NDM}-9}$, gene coding for

132 the New Delhi metallo-ß-lactamase; ble, bleomycin resistance gene, cutA, gene coding for 133 divalent-cation tolerance protein CutA: $s d b C$, gene coding for $\alpha-\mathrm{N}$-acetylgalactosaminidase; 134 trpf, gene coding for N-(5'-phosphoribosyl)anthranilate isomerase.

135

136

137 